\title{
HARMONIC POLYNOMIALS ON THE POINCARÉ DODECAHEDRAL THREE-MANIFOLD
}

\author{
PETER KRAMER
}

Communicated by Gerald A. Goldin

\begin{abstract}
With Lie-algebraic methods we obtain the set of orthogonal harmonic polynomials on the Poincaré dodecahedral three-manifold. The expansion in these polynomials of temperature fluctuations of the cosmic microwave background tests this manifold as a candidate for cosmic topology.
\end{abstract}

\section{Introduction}

The global topology of the three-space is not fixed by Einstein general relativity, since this is formulated in terms of local differential equations. Einstein's first static cosmological models used for the space-part of the universe a simply connected sphere $\mathbb{S}^{3}$. With present-day cosmological information it becomes possible to propose and test multiply-connected topologies for the space-part of the universe. J.-P. Luminet et al. [7] and J. Weeks [10] propose to explore the topology of three-space from temperature fluctuations of the cosmic microwave background $(\mathrm{CMB})$. These fluctuations are measured with very high precision.

As one way to test the topology, one can try to expand the temperature fluctuations of the CMB into harmonic polynomials of a chosen topological three-manifold, for example the Poincaré dodecahedral manifold $\mathcal{P}$. The topology will be verified if the harmonic polynomials of the manifold suffice to expand these fluctuations. We shall see that the restriction of the topology from $\mathbb{S}^{3}$ to $\mathcal{P}$ results in strong and specific selection rules for the harmonic poplynomials.

To implement such an expansion, a prerequisite is to explicitly characterize the harmonic polynomials on $\mathcal{P}$. The details of such a characterization are given in Kramer [5] and gr-qc/0410094. Here we describe the main steps of the analysis. 


\section{General Concepts from Topology}

For general notions of topology we refer to the classical monograph by Seifert and Threlfall [9]. The topology of a manifold $\mathcal{M}$ is partially characterized by its (first) homotopy group $\pi_{1}(\mathcal{M})$ [9]. This group operates on $\mathcal{M}$ by loop composition. If the manifold is multiply connected, the homotopy group is non-trivial. Associated to $\mathcal{M}$ is its simply-connected universal cover $\tilde{\mathcal{M}}$. The topological manifold $\mathcal{M}$ appears on its universal cover $\tilde{\mathcal{M}}$ in the form of a tiling into copies of $\mathcal{M}$. There is a group of deck transformations $\operatorname{deck}(\tilde{\mathcal{M}})$ [9]. It acts fixpoint-free on $\tilde{\mathcal{M}}$ and produces the tiling. This group is isomorphic to the homotopy group

$$
\operatorname{deck}(\tilde{\mathcal{M}}) \sim \pi_{1}(\mathcal{M}) .
$$

These relations allow one to work out the topology on the universal cover and to view the topological manifold $\mathcal{M}$ as the quotient space

$$
\mathcal{M}=\tilde{\mathcal{M}} / \operatorname{deck}(\tilde{\mathcal{M}})
$$

\section{Topology of the Poincaré Dodecahedral Three-manifold $\mathcal{P}$}

H. Poincaré in 1895 introduced the dodecahedral manifold $\mathcal{P}$. C. Weber and $\mathrm{H}$. Seifert in 1933 [8] gave a gluing prescription for $\mathcal{P}$ : glue all pairs of opposite faces of a dodecahedron, after rotation by $\pi / 5$, to get the topological manifold $\mathcal{P}$. H. Seifert and W. Threlfall [9] derived from this gluing prescription the homotopy group $\pi_{1}(\mathcal{P})$. Their proof requires non-trivial steps in combinatorial group theory to transform from the original gluing generators and their relations to new ones. These new generators are then shown to belong to the binary icosahedral group $\mathcal{H}_{3}$ of order $\left|\mathcal{H}_{3}\right|=120$ without reflections

$$
\operatorname{deck}(\tilde{\mathcal{P}}) \sim \pi_{1}(\mathcal{P}) \sim \mathcal{H}_{3}<\mathrm{SU}(2, \mathbb{C}) .
$$

The group $\mathcal{H}_{3}$ consists of the preimages in $\mathrm{SU}(2, \mathbb{C})$ of all the rotations of the familiar icosahedral group, which is isomorphic to the alternating group $A_{5}$ of five objects.

We now wish to view the topology on the universal cover. The Poincare dodecahedral manifold $\mathcal{P}$ has as universal cover the three-sphere, $\tilde{\mathcal{P}}=\mathbb{S}^{3}$ of constant positive curvature $\kappa=1$. By the isomorphism in (1), there is an action

$$
\operatorname{deck}(\tilde{\mathcal{P}}) \times \tilde{\mathcal{P}} \rightarrow \tilde{\mathcal{P}}
$$


such that $\tilde{\mathcal{P}}$ is tiled by images under $\mathcal{H}_{3}$ of a prototile $\mathcal{P}$. Conversely from (2), the Poincaré manifold may be taken as the quotient

$$
\mathcal{P}=\mathbb{S}^{3} / \operatorname{deck}(\tilde{\mathcal{P}})=\mathbb{S}^{3} / \mathcal{H}_{3}
$$

We shall work on the universal cover $\mathbb{S}^{3}$.

\section{Complex Coordinates and Lie Group Actions on $\mathbb{S}^{3}$}

The sphere $\mathbb{S}^{3}$ itself is a homogeneous space

$$
\mathbb{S}^{3}:=\mathrm{SO}(4, \mathbb{R}) / \mathrm{SO}(3, \mathbb{R}) .
$$

Moreover $\mathbb{S}^{3}$ as a manifold is in one-to-one correspondence with $\mathrm{SU}(2, \mathbb{C})$, so that the pair $\left(z_{1}, z_{2}\right)$ of complex numbers may serve as its coordinates, where

$$
u:=\left[\begin{array}{rr}
z_{1} & z_{2} \\
-\bar{z}_{2} & \bar{z}_{1}
\end{array}\right] \in \mathrm{SU}(2, \mathbb{C}), \quad z_{1} \bar{z}_{1}+z_{2} \bar{z}_{2}=1 .
$$

In the above coordinates $\mathbb{S}^{3}$ admits the following left and right actions:

$$
u \in \mathbb{S}^{3}, \quad\left(g_{l}, g_{r}\right) \in \mathrm{SU}(2, \mathbb{C}), \quad\left(\left(g_{l}, g_{r}\right) \times u\right)=g_{l}^{-1} u g_{r} .
$$

The left and right actions $\left(g_{l}, e\right),\left(e, g_{r}\right)$ commute. Moreover the full group $\operatorname{SO}(4, \mathbb{R})$ of isometries of $\mathbb{S}^{3}$ has the direct product form

$$
\mathrm{SO}(4, \mathbb{R})=\mathrm{SU}^{l}(2, \mathbb{C}) \times \mathrm{SU}^{r}(2, \mathbb{C}) / Z_{2}
$$

\section{Klein's Fundamental Invariant of $\mathcal{H}_{3}$}

In his monograph F. Klein [4] implements the Galois theory of $A_{5}$. He lets the binary icosahedral group $\mathcal{H}_{3}<\mathrm{SU}(2, \mathbb{C})$ act by linear fractional transforms on two complex projective coordinates $\left(z_{1}, z_{2}\right), \zeta=z_{1} / z_{2}$ as

$$
\zeta \rightarrow \zeta^{\prime}=\frac{a \zeta-\bar{b}}{b \zeta+\bar{a}}, \quad\left[\begin{array}{cc}
a & b \\
-\bar{b} & \bar{a}
\end{array}\right]=g_{r} \in \mathrm{SU}(2, \mathbb{C}), \quad a \bar{a}+b \bar{b}=1 .
$$

Rewritten in terms of $\left(z_{1}, z_{2}\right)$ equation (10) is exactly the right action in (8) of $g_{r}$ from (9) on $u \in \mathbb{S}^{3}$. Klein constructs a $\mathcal{H}_{3}$-invariant complex polynomial

$$
f_{k}\left(z_{1}, z_{2}\right):=\left(z_{1} z_{2}\right)\left[\left(z_{1} z_{1}\right)^{5}+11\left(z_{1} z_{2}\right)^{5}-\left(z_{2} z_{2}\right)^{5}\right]
$$

of degree 12 from the coordinates of the midpoints of the twelve dodecahedral faces. We shall see below that this invariant is in fact a lowest degree harmonic polynomial on $\mathcal{P}$. Moreover we shall build from this particular invariant polynomial an invariant operator-valued polynomial which quantizes any harmonic polynomial on $\mathcal{P}$. 


\section{Constructing the Group of Deck Transformations}

For the construction, we use a Coxeter group [2]. A Coxeter group is finitely generated by involutive generators and relations, encoded in a Coxeter-Dynkin diagram. The relevant spherical Coxeter group with icosahedral subgroup has the Dynkin diagram, the four involutive generators associated with the nodes of the diagram, and the non-trivial relations

$$
\begin{aligned}
\circ \stackrel{5}{-} \circ \stackrel{3}{3} \circ \stackrel{3}{-} \circ \\
\qquad:=\left\{R_{1}, R_{2}, R_{3}, R_{4} ;\left(R_{1}\right)^{2}=\left(R_{2}\right)^{2}=\left(R_{3}\right)^{2}=\left(R_{4}\right)^{2}\right. \\
\left.\quad=\left(R_{1} R_{2}\right)^{5}=\left(R_{2} R_{3}\right)^{3}=\left(R_{3} R_{4}\right)^{3}=I\right\} .
\end{aligned}
$$

Any pair of generators unlinked in the Dynkin diagram commutes. Any Coxeter group has a linear isometric representation by reflections [5] in Weyl hyperplanes, one for each generator. The first three generators in (12) form the icosahedral Coxeter group $\circ \underline{5} \circ \underline{3} \circ$. From a 3 -simplex bounded by the first three Weyl hyperplanes intersecting with $\mathbb{S}^{3}$, by Weyl reflections one can generate a dodecahedron. This dodecahedron on $\mathbb{S}^{3}$ will be identified with the prototile of (4) under the group of deck transformations. We find the following results [5]:

A1) The group $\mathcal{H}_{3}$ of deck transformations is a subgroup of the Coxeter group (12).

We construct a first Weber-Seifert gluing generator $C_{1}$ according to Figure 1 .

A2) We can express explicitly the Weber-Seifert gluing generator $C_{1}$, lifted to $\operatorname{deck}(\tilde{\mathcal{P}})$, as a product of Coxeter group elements

$$
C_{1}=R_{4} 5_{1}^{-2} \mathcal{I}=\text { even, } \quad\left\{5_{1}, \mathcal{I}\right\} \in \circ \stackrel{5}{\circ} \circ \stackrel{3}{-} \circ .
$$

Here $5_{1}$ is a five-fold rotation around the vertical axis in Figure 1, and is $\mathcal{I}$ the inversion in the center of the dodecahedron.

By conjugation of $C_{1}$ with icosahedral rotations we get five more gluings, and so find

A3) The $\operatorname{group} \operatorname{deck}(\tilde{\mathcal{P}})=\mathcal{H}_{3}$ has the subgroup embedding

$$
\mathcal{H}_{3}<S(\circ \stackrel{5}{-} \circ \stackrel{3}{-} \circ \stackrel{3}{\circ} \circ)<\mathrm{SO}(4, \mathbb{R}) .
$$

Here $S($ ) denotes the unimodular restriction of the Coxeter group to $\mathrm{SO}(4, \mathbb{R})$. 


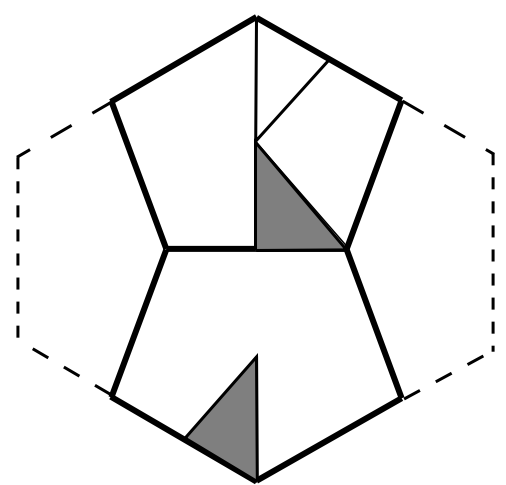

Figure 1: Gluing prescription for $\mathcal{P}$ by Weber and Seifert. Two opposite pentagonal faces of the dodecahedral prototile in a projection along a two-fold axis. 1) The inversion $\mathcal{I}$ maps the shaded triangle in the bottom pentagon face to the white triangle in the top face. 2) The operation $5_{1}^{-2}$ rotates the white triangle in the top face to the shaded position. 3 ) The Weyl generator $R_{4}$, a reflection in the top pentagon face, glues the dodecahedron to its top face neighbour.

By computing the action of $C_{1}=R_{4} 5_{1}^{-2} \mathcal{I}$ from (13) on the complex coordinates (7) for $\mathbb{S}^{3}$ we find

$$
C_{1}:\left(z_{1}, z_{2}\right) \rightarrow\left(z_{1}, z_{2}\right)(-1)\left[\begin{array}{cc}
\epsilon^{-2} & 0 \\
0 & \epsilon^{2}
\end{array}\right], \quad \epsilon:=\exp (2 \pi \mathrm{i} / 5)
$$

The matrix in (15) belongs to $\mathcal{H}_{3}$ [5].

A4) The group $\operatorname{deck}(\tilde{\mathcal{P}})=\mathcal{H}_{3}<\operatorname{SU}^{r}(2, \mathbb{C})$ acts from the right on $u \in \mathbb{S}^{3}$, and therefore commutes with the continuous left action of $\mathrm{SU}^{l}(2, \mathbb{C})$.

A5) The group $\mathcal{H}_{3}$ is normal in the unimodular restriction of the Coxeter group (12) and forms the semidirect product [5]

$$
S\left(\circ-\frac{5}{-} \circ \stackrel{3}{-} \circ \stackrel{3}{-\circ}\right)=\mathcal{H}_{3} \times{ }_{s} S\left(\circ-\frac{5}{-} \circ \stackrel{3}{-\circ}\right) \text {. }
$$

\section{From Group Actions to Representation Spaces: Harmonic Polyno- mials}

Definition 1. A harmonic polynomial $P$ on $\mathbb{S}^{3}$ is homogeneous of degree $\lambda$ and obeys $\Delta P=0$ where $\Delta$ is the Laplacian on $\mathbb{S}^{3}$.

Theorem 2. The harmonic polynomials on $\mathbb{S}^{3}$ in the complex coordinates of equation (7) are identical with Wigner's standard irreducible representations of 
$\mathrm{SU}(2, \mathbb{C})$, taken as polynomials in the elements of the matrix $u \in \mathrm{SU}(2, \mathbb{C})$ of (7) [3]

$$
P^{\lambda} \equiv D_{m, m^{\prime}}^{j}\left(z_{1}, z_{2}, \bar{z}_{1}, \bar{z}_{2}\right), \quad \lambda=2 j, \quad-j \leq\left(m, m^{\prime}\right) \leq j .
$$

Here $\lambda$ is the degree of the harmonic polynomial, $j$ is the integral or half-integral label for the irreducible representation of $\operatorname{SU}^{l}(2, \mathbb{C})$, and $\left(m, m^{\prime}\right)$ are the usual integral or half-integral row and column labels of this irreducible representation.

Proof: i) The degree of $P^{\lambda}$ is $\lambda=2 j$.

ii) For $m=j, D_{j, m^{\prime}}^{j}$ is analytic in $\left(z_{1}, z_{2}\right)$, hence $\Delta D_{j, m^{\prime}}^{j}=0$.

iii) Since $m$ can be lowered by the operator $L_{-}^{l}$ commuting with $\Delta$

$$
\Delta D_{m, m^{\prime}}^{j} \sim \Delta\left(L_{-}^{l}\right)^{j-m} D_{j, m^{\prime}}^{j}=\left(L_{-}^{l}\right)^{j-m} \Delta D_{j, m^{\prime}}^{j}=0 .
$$

The problem of finding the harmonic polynomials on $\mathcal{P}$ can now be formulated as follows:

From all the polynomials in (17), we must select the subset which belongs to the identity irreducible representation $D^{\alpha_{0}} \equiv 1$ of $\mathcal{H}_{3} \in \mathrm{SU}^{r}(2, \mathbb{C})$.

For fixed irreducible representation $j$ of $\mathrm{SU}^{r}(2, \mathbb{C})$ with character $\chi^{j}$ we can compute the multiplicity of $\mathcal{H}_{3}$-invariant polynomials from a scalar product of characters

$$
m\left(j, \alpha_{0}\right):=\frac{1}{\left|\mathcal{H}_{3}\right|} \sum_{g \in \mathcal{H}_{3}} \chi^{j}(g) \bar{\chi}^{\alpha_{0}}(g), \quad \chi^{\alpha_{0}}(g)=1
$$

with the following result:

The multiplicity $m\left(j, \alpha_{0}\right)$ of invariant harmonic polynomials is zero for degree $2 j=$ odd. For $2 j=$ even it is given by

i) the starting values $m\left(j, \alpha_{0}\right)=1$ for $j \leq 30$ are

$$
j=0,6,10,12,15,16,18,20,21,22,24,25,26,27,28
$$

and $m=0$ otherwise

ii) the recursion relation from the characters (19) is

$$
m\left(j+30, \alpha_{0}\right)=m\left(j, \alpha_{0}\right)+1 .
$$


The multiplicity (20) characterizes the subduction $\mathrm{SU}^{r}(2, \mathbb{C})>\mathcal{H}_{3}$. In addition, since $\mathcal{H}_{3}$ and $\mathrm{SU}(2, \mathbb{C})$ commute, there is for fixed $j$ an additional multiplicative degeneracy $(2 j+1)$ of harmonic polynomials, see $\mathrm{B} 4$ below.

From (20) ii) we conclude that the relative fraction up to $j$ of harmonic polynomials for $\left(\mathcal{P} / \mathbb{S}^{3}\right)$ on average is $\sum_{0}^{j} m\left(j^{\prime}, \alpha_{0}\right) /\left(\sum_{0}^{j}\left(2 j^{\prime}+1\right)\right) \sim 1 / 30$.

There are strong selection rules and a low mode suppression of harmonic polynomials on $\mathcal{P}$ versus those on its universal cover $\mathbb{S}^{3}$. Weeks [10] phrases this as the Mystery of the missing fluctuations.

\section{Group/Subgroup Subduction of Irreducible Representations by a Generalized Casimir Operator}

We proceed to the explicit determination of the invariant polynomials. For this purpose we first extend the problem and find the full group/subgroup subduction in $\mathrm{SU}(2, \mathbb{C})>\mathcal{H}_{3}$ for all irreducible representations $D^{\alpha}$ of $\mathcal{H}_{3}$, and then from these select the identity irreducible representation $D^{\alpha_{0}}$.

We follow the Bargmann and Moshinsky [1] paradigm, exemplified by them for $\mathrm{SU}(3, \mathbb{C})>\mathrm{SO}(3, \mathbb{R})$ :

Definition 3. A generalized Casimir operator $\Omega$ determines the irreducible representation subduction $G>H$ iff i), ii), iii) hold

i) $\Omega$ is from the enveloping Lie algebra $\operatorname{Env}\left(l_{G}\right)$ and so preserves irreducible representation spaces under $G$

ii) $\Omega$ is invariant under $H$ but not under $G$

iii) $\Omega$ is hermitian and non-degenerate.

Part iii) of this definition excludes for continuous $H$ its Casimir operator and for discrete $H$ its projection operators, since these cannot distinguish between repeated irreducible representations!

As tools we determine the right action generators of $\mathrm{SU}^{r}(2, \mathbb{C})$ acting on $\mathbb{S}^{3}$ according to equation (8). The right action Lie generators of $\mathrm{SU}^{r}(2, \mathbb{C})$ from (8) act on functions of $\left(z_{1}, z_{2}, \bar{z}_{1}, \bar{z}_{2}\right)$ as first order differential operators:

$$
\begin{aligned}
& L_{+}:=L_{1}^{r}+\mathrm{i} L_{2}^{r}=z_{1} \partial_{z_{2}}-\bar{z}_{2} \partial_{\bar{z}_{1}} \\
& L_{-}:=L_{1}^{r}-\mathrm{i} L_{2}^{r}=z_{2} \partial_{z_{1}}-\bar{z}_{1} \partial_{\bar{z}_{2}} \\
& L_{3}:=L_{3}^{r}=(1 / 2) z_{1} \partial_{z_{1}}-z_{2} \partial_{z_{2}}-\bar{z}_{1} \partial_{\bar{z}_{1}}+\bar{z}_{2} \partial_{\bar{z}_{2}} \\
& {\left[L_{3}, L_{ \pm}\right]= \pm L_{ \pm}, \quad\left[L_{+}, L_{-}\right]=2 L_{3} .}
\end{aligned}
$$


The left action of the Lie generators of $\mathrm{SU}^{l}(2, \mathbb{C})$ from (8) looks similar but commute with all the right Lie generators in (21) [5].

The next tool is Klein's homomorphism [4], $\mathrm{SU}^{r}(2, \mathbb{C}) \rightarrow \mathrm{SO}(3, \mathbb{R})$. Under the right action

$$
\left(z_{1}, z_{2}\right) \rightarrow\left(z_{1}, z_{2}\right) g_{r}, \quad g_{r} \in \mathrm{SU}(2, \mathbb{C})
$$

the vector

$$
((x+\mathrm{i} y) / \sqrt{2}, z,(x-\mathrm{i} y) / \sqrt{2}) \equiv\left(2 z_{1} \bar{z}_{2}, z_{1} \bar{z}_{1}-z_{2} \bar{z}_{2}, 2 \bar{z}_{1} z_{2}\right)
$$

respectively under conjugation

$$
\left(L_{+}, L_{3}, L_{-}\right) \rightarrow U_{g_{r}}\left(L_{+}, L_{3}, L_{-}\right) U_{g_{r}}^{-1}
$$

the generators from (21) transform linearly with the same representation $D^{1}\left(g_{r}\right) \in$ $\mathrm{SO}(3, \mathbb{R})$ !

The following steps are just an exercise in the noncommutative geometry of $\operatorname{Env}(\mathfrak{s u}(2, \mathbb{C}))$, similar to the Penrose length quantization in spin networks and to quantum gravity.

\section{Lie-algebraic Results on Hharmonic Polynomials of $\mathcal{P}$}

To find harmonic polynomials we proceed [5] as follows:

B1) Construct a $\mathcal{H}_{3}$-invariant polynomial

$$
\mathcal{K}^{\prime}=P\left(2 z_{1} \bar{z}_{2}, z_{1} \bar{z}_{1}-z_{2} \bar{z}_{2}, 2 \bar{z}_{1} z_{2}\right)=P((x+\mathrm{i} y) / \sqrt{2}, z,(x-\mathrm{i} y) / \sqrt{2}) .
$$

Klein's invariant polynomial from (11) can not be written in terms of the vector components in (23) which then would allow to pass to the generators $\left(L_{+}, L_{3}, L_{-}\right)$. Fortunately we can generate other invariant polynomials by applying the left lowering generator $L_{-}^{l}$ from $\mathrm{SU}^{l}(2, \mathbb{C})$ to Klein's fundamental invariant (11). Applying the power $\left(L_{-}^{l}\right)^{6}$ to Klein's invariant one obtains [5] a polynomial in which the vector components of the homomorphism (22) can be substituted

$$
\mathcal{K}^{\prime} \sim\left(\left(L_{-}^{l}\right)^{6} f_{k}\left(z_{1}, z_{2}\right) \sim(x+\mathrm{i} y)^{5} z+z(x-\mathrm{i} y)^{5}+P_{2}\left(r^{2}, z\right):=P .\right.
$$

B2) Use the Klein homomorphism (23) and substitute

$$
\mathcal{K}^{\prime}(x+\mathrm{i} y, z, x-\mathrm{i} y) \rightarrow \mathcal{K}\left(L_{+}, L_{3}, L_{-}\right) .
$$


Since now we are dealing with homogeneous polynomials of degree $n$, we run into the noncommutativity problem of operator-valued polynomials in $\left(L_{+}, L_{3}, L_{-}\right)$. To assure the same transformation of the polynomial operator $\mathcal{K}$ as for $\mathcal{K}^{\prime}$, we must apply the operator of symmetrization defined for polynomial operators $P$ by

$$
\operatorname{Sym} P\left(A_{1}, \ldots, A_{n}\right):=\frac{1}{n !} \sum_{\left(i_{1}, \ldots, i_{n}\right) \in S_{n}} P\left(A_{i_{1}}, \ldots, A_{i_{n}}\right) .
$$

In this way we get from equations (26) and (27) the $\mathcal{H}_{3}$-invariant hermitian generalized Casimir operator

$$
\begin{aligned}
\mathcal{K} & :=\operatorname{Sym}\left[\mathcal{K}\left(L_{+}, L_{3}, L_{-}\right)\right] \in \operatorname{Env}\left(\operatorname{SU}^{r}(2, \mathbb{C})\right) \\
& =\operatorname{Sym}\left[\left(L_{+}\right)^{5} L_{3}+L_{3}\left(L_{-}\right)^{5}+P_{2}\left(L^{2}, L_{3}\right)\right] .
\end{aligned}
$$

By construction $\mathcal{K}$ commutes with the Casimir operator $\Lambda^{2}$ of $\operatorname{SO}(4, \mathbb{R})$. Note that Sym has $6 !=720$ terms. The symmetrization (28) is performed in the appendix of [5].

B3) Quantize the spherical harmonics $P^{2 j}$ by diagonalizing the right action of $\mathcal{K}$. The eigenspaces are characterized by irreducible representations $D^{\alpha}$ of $\mathcal{H}_{3}$.

B4) $\mathcal{K}$ commutes with $\mathrm{SU}(2, \mathbb{C})$, so the degeneracy of any real eigenvalue $\kappa$ of $\mathcal{K}$ is $(2 j+1) \operatorname{dim}(\alpha)$, and the harmonic polynomials on $\mathbb{S}^{3}$ are now characterized by

$$
\begin{aligned}
& P=P_{m, \kappa}^{2 j}\left(z_{1}, z_{2}, \bar{z}_{1}, \bar{z}_{2}\right), \quad \Delta P_{m, \kappa}^{2 j}=0, \quad(x \cdot \nabla) P_{m, \kappa}^{2 j}=(2 j) P_{m, \kappa}^{2 j} \\
& \Lambda^{2} P_{m, \kappa}^{2 j}=4 j(j+1) P_{m, \kappa}^{2 j}, \quad \mathcal{K} P_{m, \kappa}^{2 j}=\kappa P_{m, \kappa}^{2 j}, \quad L_{3}^{l} P_{m, \kappa}^{2 j}=m P_{m, \kappa}^{2 j} .
\end{aligned}
$$

B5) Harmonic polynomials on $\mathcal{P}$ must belong to the identity irreducible representation $\alpha_{0}$ of $\mathcal{H}_{3}$.

B6) The Spectrum of $\mathcal{K}$ ( [5] pp. 3530-1): $\mathcal{K}$ by (28) acts in linear subspaces $\mathcal{L}^{\mu}: m \equiv \mu \bmod 5$ and in these subspaces is tridiagonal. By general reasons given in [1], it follows from tridiagonality that the operator $\mathcal{K}$ in any linear subspace $\mathcal{L}^{\mu}$ is non-degenerate (no repeated eigenvalues). Moreover, it is shown in ([5] p.3531) that the identity irreducible representation $D^{\alpha_{0}}$ of $\mathcal{H}_{3}$ can occur only in $\mathcal{L}^{0}$.

The hermiticity of $\mathcal{K}$ yields the orthogonality of the eigenstates. 
Theorem 4. The properties $\mathrm{B} 1-\mathrm{B} 6$ taken together show that the operator $\mathcal{K}$ by its eigenvalues and eigenstates completely characterizes the harmonic polynomials on the dodecahedral Poincaré manifold $\mathcal{P}$. Moreover, the unique characterization by $\mathcal{K}$ of the subduction in $\mathrm{SO}(4, \mathbb{R})>\mathcal{H}_{3}$ holds true for any irreducible representation $D^{\alpha}$ of $\mathcal{H}_{3}[5]$.

As an example for the diagonalization of $\mathcal{K}$ we consider the case $j=6$. The values of $\mu$ and corresponding values of $m$ are given by

$$
\begin{array}{lllllll}
j=6 & \mu: & 0 & 1 & 2 & 3 & 4 \\
& m: & (-5,0,5) & (-4,1,6) & (-3,2) & (-2,3) & (-6,-1,4)
\end{array}
$$

From B6, we expect harmonic polynomials only in $\mathcal{L}^{0}$ of dimension three. Evaluation of the operator $\mathcal{K}$ in this subspace gives its matrix form and eigenvalues

$$
\begin{aligned}
\mathcal{K} V^{\mu} & =V^{\mu} \mathcal{K}^{\mu, \text { diag }} \\
j & =6, \quad \mu=0, \quad m=(-5,0,5) \\
\kappa & =\left(-51975,-\frac{51975}{2}, \frac{14175}{2}\right) \\
\mathcal{K} & =\left[\begin{array}{rrr}
-\frac{51975}{2} & \frac{4725 \sqrt{77}}{2} & 0 \\
\frac{4725 \sqrt{77}}{2} & -18900 & -\frac{4725 \sqrt{77}}{2} \\
0 & -\frac{4725 \sqrt{77}}{2} & -\frac{51975}{2}
\end{array}\right] \\
V^{0} & =\left[\begin{array}{rrr}
-\sqrt{\frac{7}{25}} & \sqrt{\frac{1}{2}} & -\sqrt{\frac{11}{50}} \\
\sqrt{\frac{11}{25}} & 0 & -2 \sqrt{\frac{7}{50}} \\
\sqrt{\frac{7}{25}} & \sqrt{\frac{1}{2}} & \sqrt{\frac{11}{50}}
\end{array}\right] \\
\mathcal{K}^{0, \text { diag }} & =\left[\begin{array}{ccc}
-51975 & \\
& -\frac{51975}{2} & \frac{14175}{2}
\end{array}\right] .
\end{aligned}
$$

The first eigenstate with $\kappa=-51975$ is a harmonic polynomial and up to a constant factor turns out to be Klein's $f_{k}\left(z_{1}, z_{2}\right)$ from (11)! Twelve more orthogonal companions with the same eigenvalue $\kappa$ are obtained by applying powers of the lowering operator $L_{-}^{l}$.

The other two eigenstates in (31) are not harmonic polynomials for the following reason: It turns out ( [5] p. 3534) that they have companions with the same eigenvalue $\kappa$, but in subspaces $\mathcal{L}^{\mu}, \mu \neq 0$. Together with these they span irreducible 
representations $D^{\alpha}, \alpha \neq \alpha_{0}$ of $\mathcal{H}_{3}$ and therefore are not harmonic polynomials of $\mathcal{P}$.

The explicit diagonalization of $\mathcal{K}$ for degrees $2 j \leq 12$ is given in ( [5] pp. 35323536). There is no problem in going on to any higher degree harmonic polynomials as eigenstates of $\mathcal{K}$. Since these harmonic polynomials are orthogonal, the expansion coefficients of the observed CMB fluctuations in terms of harmonic polynomials are given by the scalar products between the observed fluctuations and these polynomials. The strict validity of the Poincare manifold as a model for the space-part of the cosmos would imply that all scalar products beween the fluctuations and harmonic polynomials not belonging to the identity irreducible representation $D^{\alpha_{0}}$ of $\mathcal{H}_{3}$ must vanish.

\section{Conclusions}

- The subduction $\mathrm{SO}(4, \mathbb{R})>\mathcal{H}_{3}$ for any irreducible representation of $\mathcal{H}_{3}$ is explicitly resolved by the operator $\mathcal{K}$. Harmonic polynomials on $\mathcal{P}$ become (non-degenerate) eigenstates of $\mathcal{K}$.

- For degree $2 j=12$, only Felix Klein's invariant harmonic polynomial $f_{k}$ in (11) plus twelve orthogonal companions belong to the non-degenerate eigenvalue $\kappa=-51975$ of $\mathcal{K}$. All other eigenstates have degenerate companions in subspaces $\mathcal{L}^{\mu^{\prime}}, \mu^{\prime} \neq 0$ belonging to irreducible representations $\alpha \neq \alpha_{0}$.

- There is an additional controlled degeneracy $(2 j+1)$ from invariance under $\mathrm{SU}^{l}(2, \mathbb{C})$.

- If three-space has the topology of $\mathcal{P}$, we can expand the temperature fluctuations of CMB exclusively in invariant eigenmodes of $\mathcal{K}$.

- A similar analysis can be done for the topological three-manifolds $\mathbb{S}^{3} / T^{*}$, $\mathbb{S}^{3} / O^{*}$. with $T^{*}, O^{*}$ the binary tetrahedral, octahedral group. All these three-manifolds share $\mathbb{S}^{3}$ as their universal cover.

- What about hyperbolic three-manifolds [6]? Here the universal cover is the hyperbolic space of dimension three. 


\section{References}

[1] Bargmann V. and Moshinsky M., Group Theory of Harmonic Oscillators I: The Collective Modes, Nucl. Phys. 18 (1960) 697-712, II: The Integrals of Motion of the Quadrupole-quadrupole Interaction, Nucl Phys. 23 (1961) 177-199.

[2] Coxeter H., Regular Polytopes, Dover, New York, 1973.

[3] Edmonds A., Angular Momentum in Quantum Mechanics, Princeton University Press, Princeton, 1957.

[4] Klein F., Vorlesungen über das Ikosaeder, B. G. Teubner, Leipzig 1884, Reprint Ed. P. Slodowy, Birkhäuser, Basel, 1993.

[5] Kramer P., An Invariant Operator Due to F. Klein Quantizes H. Poincaré's Dodecahedral Three-manifold, J. Phys. A 38 (2005) 3517-3540.

[6] Kramer P., Group Actions on Compact Hyperbolic Manifolds and Closed Geodesics, In: Symmetry in Physics, CRM Proc. and Lecture Notes vol. 34, P. Winternitz et al. (Eds.), AMS, Providence, 2004, pp. 113-124.

[7] Luminet J.-P., Weeks J., Riazuelo A., Lehoucq R. and Uzan J.-Ph., Dodecahedral Space Topology as an Explanation for Weak Wide-angle Temperature Correlations in the Cosmic Microwave Background, Nature 425 (2003) 593595.

[8] Weber C. and Seifert H., Die beiden Dodekaederräume, Math. Z. 37 (1933) 237-253.

[9] Seifert H. and Threlfall W., Lehrbuch der Topologie, Leipzig 1934, Reprint Chelsea Publ. Comp., New York, 1980.

[10] Weeks J., The Poincaré Dodecahedral space and the Mystery of the Missing Fluctuations, Notices of the AMS 51 (2004) 610-619.

Peter Kramer

Institut für Theoretische Physik

Universität Tübingen

72076 Tübingen, GERMANY

E-mail address: peter.krameruni-tuebingen.de 